\title{
First-principles study of the dynamic Jahn-Teller distortion of the neutral vacancy in diamond
}

\author{
Joseph C. A. Prentice, ${ }^{1}$ Bartomeu Monserrat, ${ }^{1,2}$ and R. J. Needs ${ }^{1}$ \\ ${ }^{1}$ TCM Group, Cavendish Laboratory, University of Cambridge, J. J. Thomson Avenue, Cambridge CB3 OHE, United Kingdom \\ ${ }^{2}$ Department of Physics and Astronomy, Rutgers University, Piscataway, New Jersey 08854-8019, USA
}

(Received 7 October 2016; published 20 January 2017)

\begin{abstract}
First-principles density functional theory methods are used to investigate the structure, energetics, and vibrational motions of the neutral vacancy defect in diamond. The measured optical absorption spectrum demonstrates that the tetrahedral $T_{d}$ point group symmetry of pristine diamond is maintained when a vacancy defect is present. This is shown to arise from the presence of a dynamic Jahn-Teller distortion that is stabilized by large vibrational anharmonicity. Our calculations further demonstrate that the dynamic Jahn-Teller-distorted structure of $T_{d}$ symmetry is lower in energy than the static Jahn-Teller distorted tetragonal $D_{2 d}$ vacancy defect, in agreement with experimental observations. The tetrahedral vacancy structure becomes more stable with respect to the tetragonal structure by increasing temperature. The large anharmonicity arises mainly from quartic vibrations, and is associated with a saddle point of the Born-Oppenheimer surface and a minimum in the free energy. This study demonstrates that the behavior of Jahn-Teller distortions of point defects can be calculated accurately using anharmonic vibrational methods. Our work will open the way for first-principles treatments of dynamic Jahn-Teller systems in condensed matter.
\end{abstract}

DOI: 10.1103/PhysRevB.95.014108

\section{INTRODUCTION}

Point defects in crystals introduce electron energy levels within the electronic band gap, trap charge carriers, and emit and absorb light, and phonon scattering from them limits thermal and electrical conductivities [1,2]. Such effects influence many of the most desirable properties of diamond, including its optical properties and high thermal conductivity, and point defects in diamond have been the subject of much previous theoretical work $[3,4]$. Some defects such as the $\mathrm{Si}-\mathrm{V}[5,6]$ and $\mathrm{N}-\mathrm{V}^{-}$centers [7] have been identified as potential "qubits" in quantum computers [2,8-13]. Both these defects include a lattice vacancy, which is an important defect in its own right. The neutral vacancy in diamond is particularly significant because it is known to be stable over a wide range of doping levels [14] and plays a central role in defect diffusion [15]. It is also important for its optical absorption and luminescence properties - it is associated with a series of lines in the absorption spectrum of diamond, including the strong and sharp GR1 line at $1.673 \mathrm{eV} \mathrm{[16]—and} \mathrm{its} \mathrm{applications} \mathrm{in}$ quantum information [17] and precision sensing [18].

Carbon and silicon are isoelectronic, and their pristine lattices have tetrahedral $T_{d}$ point group symmetry, as depicted in Fig. 1(a). Watkins modeled the neutral vacancy in silicon using a linear combination of atomic orbitals including only the four atoms surrounding the vacancy [19], showing that two electrons should occupy a triply degenerate state. The energy of these electrons can be lowered by splitting this degenerate energy state via a static Jahn-Teller distortion $[19,20]$ of tetragonal $D_{2 d}$ symmetry [14,21,22]. Theoretically, the presence of a Jahn-Teller distortion is revealed by the emergence in the undistorted structure of harmonic vibrational soft modes, i.e., modes with imaginary frequencies; this approach successfully predicts the $D_{2 d}$ distortion experimentally observed in the silicon vacancy. When applied to diamond, this approach also predicts a $D_{2 d}$ distortion, but in this case the experimental observations show that the neutral diamond vacancy has $T_{d}$ symmetry instead $[23,24]$. [Vacancy structures with both symmetries are shown in Figs. 1(b) and 1(c).] The experimental observations can be rationalized by the appearance of a dynamic Jahn-Teller distortion [22,23,25,26], due to strong anharmonic vibrational motion. The dynamic Jahn-Teller effect is observed in a variety of systems, including doped manganites [27,28], fullerides [29,30], octahedral complexes of $d^{9}$ ions [31], and the excited states of the $\mathrm{N}-\mathrm{V}^{-}$center in diamond [32].

In a dynamic Jahn-Teller system, there are two or more minima in the Born-Oppenheimer (BO) energy surface, which are separated by energy barriers. In the vacancy in diamond, there are three minima, corresponding to tetragonal distortions in each Cartesian direction. If the energy barriers between the minima are low enough, the wave function of the system is shared between the minima instead of localizing in a single minimum, resulting in a dynamic Jahn-Teller effect. This means that the vibrational wave function possesses the symmetry of the undistorted structure [26]. Structures at saddle points or maxima of the BO surface can be stabilized by anharmonic vibrations, even at zero temperature, through zeropoint motion. Previous estimates, from experimental data, of the energy barriers between minima and an Einstein-like frequency for the tetragonal defect modes of the vacancy in diamond show that the vibrational energy quantum is larger than or comparable to the barrier energy, implying a dynamic Jahn-Teller effect close to $0 \mathrm{~K}$ [33].

The dynamic Jahn-Teller effect in the neutral vacancy in diamond is well established experimentally, but from a first-principles standpoint the view is less clear. The commonly used harmonic approximation for lattice dynamics cannot account for the presence of a dynamic Jahn-Teller distortion, as this is an intrinsically anharmonic effect. First-principles calculations of the anharmonic vibrational wave function of the ground state of the neutral vacancy in diamond have not been reported previously, meaning that a proper description of the dynamic Jahn-Teller effect in this system is lacking. In this study, we determine for the first time an anharmonic wave function which accurately describes the dynamic Jahn- 


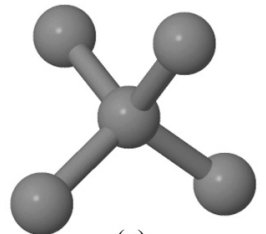

(a)

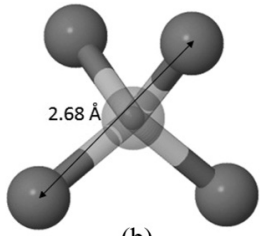

(b)

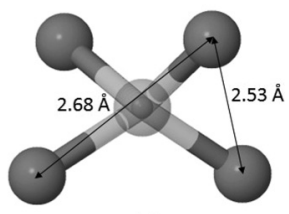

(c)
FIG. 1. Structure of the pristine diamond lattice and possible distortions of the vacancy. (a) shows a site in the pristine lattice and its four nearest neighbors. (b) shows the nearest neighbors of the vacancy with a distortion of $T_{d}$ symmetry, and (c) shows the vacancy structure with $D_{2 d}$ symmetry. The lengths indicate the distances between atoms in the relaxed structures. The four atoms in (c) form two pairs.

Teller distortion, providing a theoretical description of the experimentally observed $T_{d}$ symmetry of the neutral vacancy in diamond.

We have used first-principles DFT methods [34] to calculate the electronic and vibrational properties of the neutral vacancy. The electronic ground state is expected to be well described within DFT, although some of its electronic excited states are not because they have many-body (multideterminant) character. Our study is confined to the electronic ground state. The vibrational calculations were carried out using a recently proposed vibrational self-consistent field (VSCF) method [35], which neglects nonadiabatic effects but includes anharmonicity.

We find that formation of the dynamic Jahn-Teller $T_{d}$ vacancy structure leads to a small reduction in the vibrational energy compared with the static Jahn-Teller $D_{2 d}$ structure when anharmonic effects are included. Our results show that anharmonic nuclear motion leads to a dynamic Jahn-Teller distortion of the vacancy in diamond very close to zero temperature, and we find that this remains true at least up to $400 \mathrm{~K}$.

The rest of this work is organized as follows: In Sec. II we outline the computational methods employed and give technical details of the calculations. In Sec. III we present the main results of our study, detailing the effects of including harmonic and anharmonic contributions to the total energy, and to the vibrational density of states in each symmetry state. In Sec. IV we give a brief summary of our results and some concluding remarks.

\section{CALCULATIONAL METHODS}

\section{A. Electronic calculations}

DFT calculations were performed with version 7.0.3 of the CASTEP code [36] and the corresponding "on-the-fly" ultrasoft carbon pseudopotential [37]. We have used the local density approximation (LDA) as parametrized by Perdew and Zunger [38], which has been widely used in previous calculations involving diamond and similar materials [14,21,39-41]. LDA-DFT calculations provide a lattice constant for diamond [34] of $3.529 \AA$, compared to the experimental value of $3.567 \AA$ [42]. In our calculations, the LDA-DFT lattice constant is generally used, although some calculations were also carried out using the experimental lattice constant to investigate the effect on the dynamic stability of the tetrahedral symmetry state. Increasing the lattice constant was found to increase the stability.

To ensure the existence of a locally stable tetragonal state (i.e., one that does not relax back to the tetrahedral symmetry when geometry-optimized), a 256-atom (255 when the vacancy is present) supercell is used throughout this work. In previous DFT work on the neutral vacancy in silicon, it was observed that supercells of at least 256 atoms were required to obtain a stable tetragonal distortion $[14,21]$, constructed as a $2 \times 2 \times 2$ supercell of a 32 -atom bcc unit cell. Calculations with supercells with less than 256 atoms show that the same is true for diamond. The 256-atom supercells also allow us to effectively isolate the periodic defects from one another so that accurate structures for the tetrahedral and tetragonal vacancies can be obtained.

The geometry was optimized such that the root mean square of the forces on all of the atoms was below $0.001 \mathrm{eV} / \AA$. A simple 2-atom unit cell was relaxed to obtain the relaxed LDA-DFT lattice constant, and a 256-atom supercell was constructed from this. A vacancy was then created and the internal coordinates relaxed. The tetrahedral state was found by imposing the pristine lattice symmetry before relaxing, and the tetragonal state was found by imposing a tetragonal distortion on the four nearest neighbors of the vacancy, before relaxing with no symmetry constraints. In both vacancy structures, the nearest neighbors relaxed away from the vacancy (by $0.11 \AA$ in the tetrahedral case) in order to increase their $s p^{2}$-like bonding, as observed in previous work [22].

A plane-wave cutoff energy of $650 \mathrm{eV}$ was used for relaxing the structures and for the harmonic vibrational calculations, with a $5 \times 5 \times 5$ Monkhorst-Pack k-point grid [43], as the energy differences between the structures were very well converged for these parameters. A larger value of the cutoff energy was used for some calculations, corresponding to even stricter convergence criteria. The SCF cycle threshold for the energy was taken to be $10^{-10} \mathrm{eV}$ per atom to ensure a very accurate charge density, and thus accurate forces.

\section{B. Vibrational calculations}

A finite-displacement method was used to obtain the matrix of force constants, which was Fourier transformed to obtain the dynamical matrix [44]. This was diagonalized to obtain the harmonic vibrational frequencies and eigenvectors. Atomic displacements of $0.00529 \AA$ were used. The anharmonic vibrational calculations were conducted using the VSCF method described in Ref. [35] and used successfully several times since [45-47].

In this method, the BO energy surface is described in a basis of harmonic normal coordinates of amplitude $u$. In these coordinates, the harmonic potential is separable, and each degree of freedom contributes $\frac{1}{2} \omega^{2} u^{2}$, where $\omega$ is a harmonic frequency. Starting from the harmonic approximation, we improve the representation of the BO surface by using a principal-axes approximation [48], which takes the form of a sum over many-body terms, truncated at second order:

$$
E(\mathbf{u})=E(\mathbf{0})+\sum_{i} V_{1}\left(u_{i}\right)+\frac{1}{2} \sum_{i^{\prime} \neq i} V_{2}\left(u_{i}, u_{i^{\prime}}\right) .
$$


In this expression, $\mathbf{u}$ is a vector containing the normal-mode amplitudes $u_{i}$, Cartesian indices are collective labels for the quantum numbers $(\mathbf{q}, v)$ where $\mathbf{q}$ is a phonon wave vector and $v$ a phonon branch, and $E(\mathbf{u})$ is the value of the $\mathrm{BO}$ energy surface when the atomic nuclei are in configuration u. Anharmonicity is already included in the $V_{1}$ terms, as they are not constrained to the harmonic form. The $V_{2}$ terms provide additional anharmonic corrections arising from the 2-dimensional subspaces of the BO energy surface that they span. The terms $V_{1}$ and $V_{2}$ in this expansion are found by mapping the $\mathrm{BO}$ energy surface as a function of the amplitude $u$ of each normal mode, using a series of DFT calculations and then fitting cubic splines to the results. In this work, 19 different amplitudes per mode are used in mapping the BO surface. Mapping the BO surface is by far the most computationally expensive part of the entire calculation; for example, mapping the $V_{1}$ terms scales as the number of modes multiplied by the number of mapping points $n$ multiplied by the cost of a DFT calculation, which scales asymptotically as $n N^{4}$ for a simulation cell with $N$ atoms. The resulting anharmonic nuclear Schrödinger equation is solved using the VSCF method to give the anharmonic vibrational energy and free energy. The anharmonic vibrational wave function $|\Phi(\mathbf{u})\rangle$ is written as a Hartree product of the normal modes, $\prod_{i}\left|\phi_{i}\left(u_{i}\right)\right\rangle$. The states $\left|\phi_{i}\left(u_{i}\right)\right\rangle$ are represented in terms of basis functions which are the eigenstates of one-dimensional harmonic oscillators. A total of 40 basis functions were used for each degree of freedom.

In most of this work only the $V_{1}$ terms are included in the expansion of $E(\mathbf{u})$. The number of calculations required to map the BO surface accurately as a function of two normal coordinates is approximately the square of the number required for one coordinate, making this computationally unfeasible if all pairs of modes were to be included. Our tests show that the $V_{2}$ terms are small for most modes, as the harmonic approximation works well for vibrations in diamond [35]. The only large $V_{2}$ term corresponds to the 2-dimensional $\mathrm{BO}$ subspace spanned by two soft modes $\left(u_{1}, u_{2}\right)$ that are present in the tetrahedral structure of the diamond vacancy. This $V_{2}$ term has three equivalent minima, corresponding to a tetragonal distortion along each Cartesian direction. No matter how the orthogonal normal coordinates $\left(u_{1}, u_{2}\right)$ are chosen, it is not possible for the axes to pass precisely through more than one of these minima. This means that it is impossible to capture the behavior of the system with 3 minima using only the $V_{1}$ terms for these soft modes; the associated $V_{2}$ term must therefore be included. Furthermore, the vibrational wave function for this $V_{2}$ subspace of the BO surface cannot be described correctly as a product of two states labeled by the two corresponding normal modes $\left(u_{1}, u_{2}\right)$. Separating the wave function in this way breaks the rotational symmetry of the problem. Instead, for this subspace, we separate the wave function using polar coordinates, $r_{u}=\sqrt{u_{1}^{2}+u_{2}^{2}}$ and $\theta_{u}=\arctan \left(u_{2} / u_{1}\right)$, where the $u_{i}$ are written in units of $1 / \sqrt{2\left|\omega_{i, s}\right|}$, with $\omega_{i, s}$ the imaginary harmonic soft phonon frequencies. This preserves the correct symmetry of the problem and allows an accurate wave function to be determined. The usual wave function $\left|\phi_{1}\left(u_{1}\right)\right\rangle\left|\phi_{2}\left(u_{2}\right)\right\rangle$ is reexpressed as $\left|R\left(r_{u}\right)\right\rangle\left|T\left(\theta_{u}\right)\right\rangle$, where $\left|R\left(r_{u}\right)\right\rangle$ is written as a sum of isotropic harmonic oscillator radial basis states, and
$\left|T\left(\theta_{u}\right)\right\rangle$ is a sum of sinusoids. A total of 80 angular basis functions and 20 radial basis functions are used for these calculations. As all of the other modes are almost harmonic and therefore well described by the $V_{1}$ terms, the energy contribution from this particular $V_{2}$ subspace can simply be added to the energy of the rest of the $V_{1}$ terms of the tetrahedral defect.

It would be extremely computationally expensive to map all 762 anharmonic modes for both the tetrahedral and the tetragonal symmetry states of the vacancy, even if only the $V_{1}$ terms were included. However, we can take advantage of the small anharmonicity in pristine diamond [35]. The fact that pristine diamond is well described by the harmonic approximation implies that a necessary condition for modes to have significant anharmonic character is that they are strongly affected by the presence of the vacancy. We expect that these modes will generally have short wavelengths and high energies, as over longer length scales the effect of the vacancy will be averaged out. The effect of the vacancy on each mode can be calculated as the difference between the vacancy harmonic vibrational density of states (vDoS) and the pristine vDoS at the frequency of each mode, $\Delta g(\omega)=$ $g_{\mathrm{vib}}^{\mathrm{vac}}(\omega)-g_{\mathrm{vib}}^{\text {pris }}(\omega)$. We can then obtain an accurate value for the anharmonic correction to the energy without having to map all 762 modes by simply mapping the modes in descending order of $\Delta g(\omega)$, using the harmonic approximation for all unmapped modes, and converging the anharmonic correction with respect to the number of modes mapped. Converging the correction to within $0.1 \mathrm{meV}$ requires 32 modes to be mapped for the tetrahedral state, but 350 for the tetragonal state, implying that the distortion away from tetrahedral symmetry leads to a large increase in anharmonicity.

To further reduce the computational expense of the anharmonic calculations, an energy cutoff of $350 \mathrm{eV}$ was used, with a $5 \times 5 \times 5$ Monkhorst-Pack k-point grid [43], as the shape of the BO surface is well converged for these parameters. In some calculations larger values of these parameters were used, corresponding to even stricter convergence criteria. The energy differences between structures were converged to within $0.5 \mathrm{meV}$ per atom, with the self-consistency energy threshold for the DFT calculations set to $10^{-6} \mathrm{eV}$ per atom. The anharmonic correction to the energy in the pristine 256-atom supercell was calculated from the anharmonic correction for a 16-atom fcc supercell.

\section{RESULTS}

\section{A. Jahn-Teller effect and dynamical stability}

Figure 2 shows the calculated electronic density of states (eDoS) for the pristine, tetrahedral, and tetragonal vacancy structures. There is little difference between the eDoS of the pristine and vacancy states, apart from the appearance of a peak in the band gap almost exactly at the Fermi level in the vacancy structures. This state arises from the four "dangling bonds" around the vacancy, as predicted by the Watkins model. The inset to Fig. 2 shows that the peak splits into two upon introduction of the tetragonal distortion, as would be expected for a static Jahn-Teller distortion. These peaks correspond to the singlet and doublet states predicted by the Watkins model [19]. 


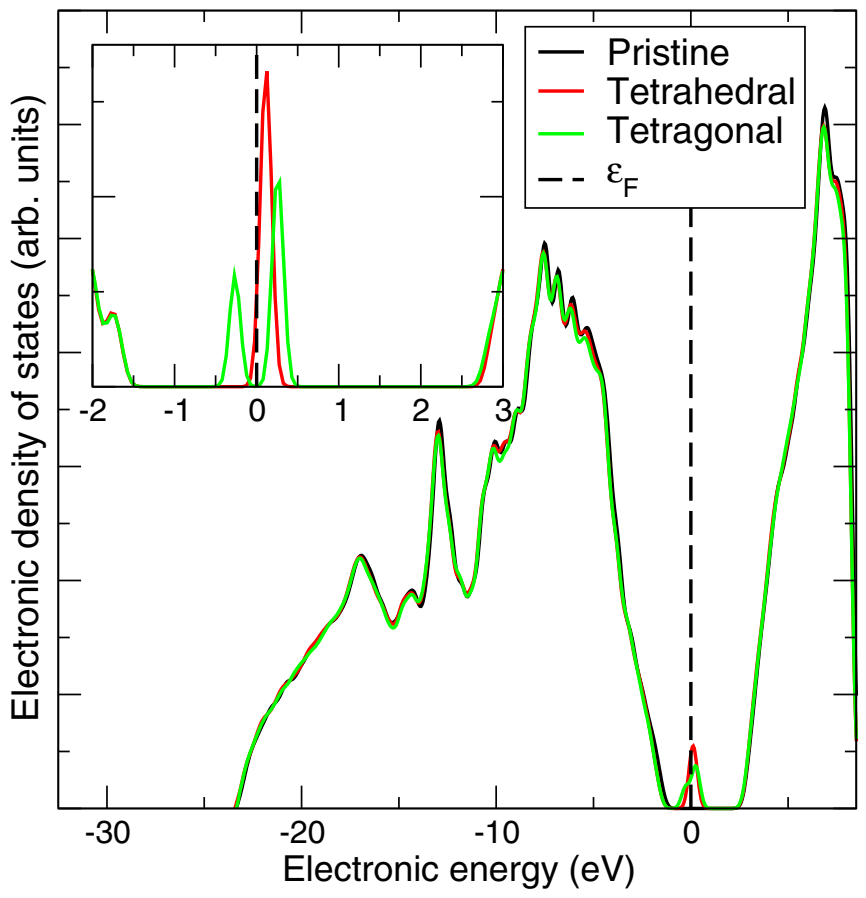

FIG. 2. Electronic density of states of diamond. The dotted line marks the Fermi energy, $\varepsilon_{\mathrm{F}}$. The inset contains the eDoS of the vacancy gap state, showing that the tetragonal distortion splits the state into a singlet at around $-0.3 \mathrm{eV}$ and a doublet at around $0.3 \mathrm{eV}$.

Harmonic calculations show that the tetrahedral state is dynamically unstable, that is, it exhibits two soft modes, with frequencies $\omega_{1, s}$ and $\omega_{2, s}$, that correspond to tetragonal distortions. Unlike the tetrahedral symmetry state, the tetragonal configuration is dynamically stable, leading to the conclusion that, at this level of theory, a static Jahn-Teller distortion of tetragonal symmetry is favored.

Including anharmonicity in the treatment of the tetrahedral configuration provides a very different picture. Figure 3 shows

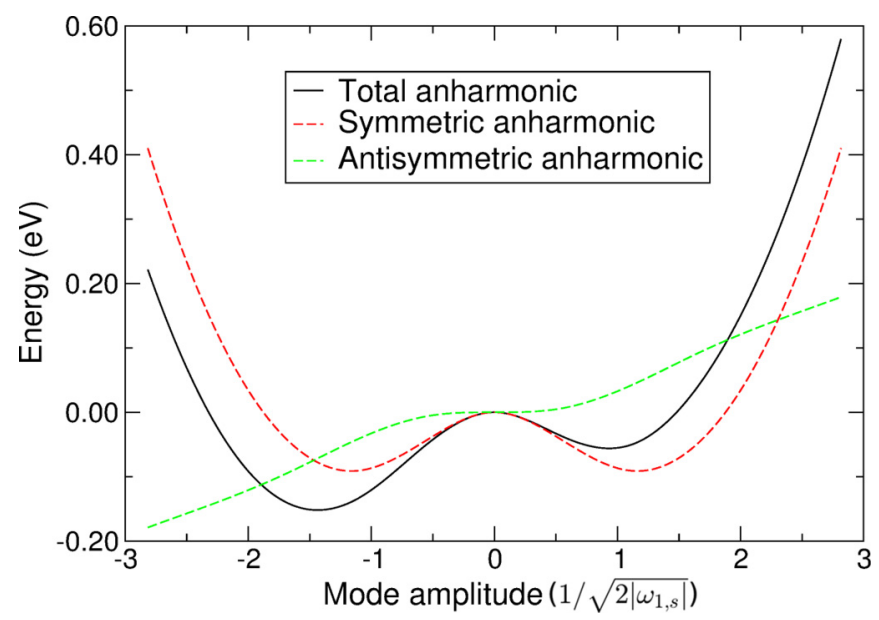

FIG. 3. Anharmonic Born-Oppenheimer energy surface mapped along the direction of one of the two soft modes of the tetrahedral structure, as well as its decomposition into symmetric and antisymmetric parts. The symmetric part of the anharmonic energy surface is clearly quartic, giving two minima.
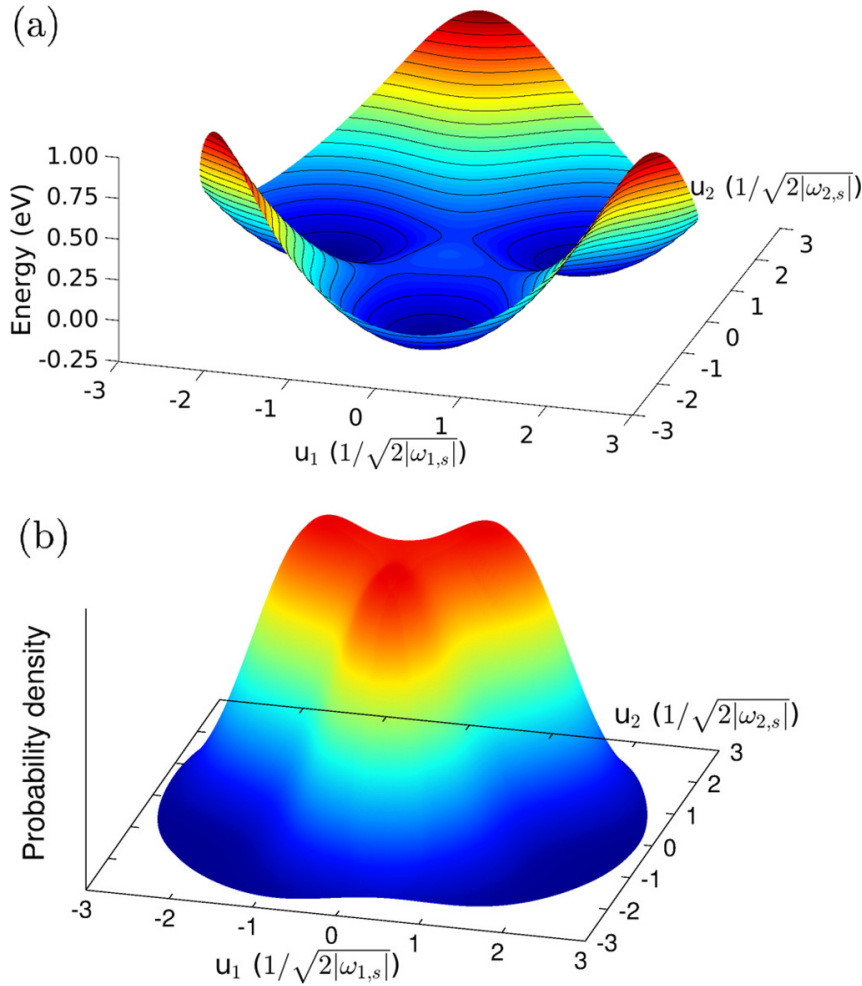

FIG. 4. (a) Born-Oppenheimer energy surface in the plane spanned by the two soft modes of the tetrahedral structure. The static tetrahedral structure lies on the local maximum at the origin. Three equivalent minima corresponding to the three possible tetragonal distortions are arranged symmetrically around the tetrahedral structure. Each contour line represents an energy increase of $0.0615 \mathrm{eV}$. (b) Anharmonic vibrational ground state probability density as a function of the amplitudes of the soft modes of the tetrahedral structure. The density has three peaks that correspond to the minima of the $\mathrm{BO}$ surface in (a).

the BO surface mapped along one of the soft mode directions, split into symmetric and antisymmetric contributions to the anharmonic energy surface. The antisymmetric part mostly arises due to the fact that any given direction cannot pass through two minima and the origin, meaning that both minima cannot be well mapped by a one-dimensional slice, as discussed above. However, the symmetric contribution clearly shows that quartic anharmonicity is present. Due to the impossibility of describing all three minima correctly using one-dimensional subspaces spanned by either soft mode individually, it is necessary to include the full 2-dimensional subspace spanned by both soft modes. Figure 4(a) shows the $V_{2}$ term of the BO surface mapped on the plane spanned by the two soft modes, and Fig. 4(b) shows the anharmonic ground state nuclear density in this subspace. In addition to the slice shown in Fig. 3, further slices through the BO surface in Fig. 4(a) can be found in the Supplemental Material [49]. The anharmonic ground state vibrational density of the tetrahedral structure has peaks in each of the three minima of the BO surface, which lowers the overall energy of the system. The fact that the wave function is shared between the minima shows that, when anharmonic vibrational effects are included, the Jahn-Teller effect in this system becomes dynamic rather than 
static, with the system maintaining the full $T_{d}$ point symmetry of the pristine lattice.

The dynamical stability of the tetrahedral state is somewhat sensitive to the exact form of the BO surface found in the DFT calculations. If the relaxed LDA lattice constant (3.529 $\AA$ ) is used when mapping the 2-dimensional BO surface of the pair of soft modes, the tetrahedral state is still dynamically unstable at $0 \mathrm{~K}$, becoming stable at $16.9 \mathrm{~K}$. However, using the experimental lattice constant of $3.567 \AA$ [42] reduces the size of the dynamical instability significantly, decreasing the absolute value of the already small ground state energy associated with the $V_{2}$ subspace by an order of magnitude. Using the experimental lattice constant the tetrahedral state is calculated to become dynamically stable at $8.6 \mathrm{~K}$. Given the errors inherent in DFT calculations, our results are consistent with the tetrahedral state being dynamically stable down to liquid helium temperatures, as implied by experiment [25], and even to absolute zero.

The minima in the BO surface are, in the polar coordinates defined above, at $r_{u}=1.64 / \sqrt{2\left|\omega_{s}\right|}, \theta_{u}=39^{\circ}, 159^{\circ}, 279^{\circ}$, which correspond to tetragonal distortions along the $x, z$, and $y$ directions, respectively. $\omega_{s}=\left(\omega_{1, s}+\omega_{2, s}\right) / 2 \simeq \omega_{1, s} \simeq \omega_{2, s}$. The values of $\theta_{u}$ depend on the precise choice of the axes defined by the two soft modes. The displacement patterns corresponding to the modes $u_{1}$ and $u_{2}$ in this work are presented in the Supplemental Material [49], allowing these minima to be unambiguously identified. At these minima, the four nearest neighbors of the vacancy are displaced from their tetrahedral equilibrium positions; they are displaced by $0.074 \AA$ away from the vacancy along the distortion direction, but by half this distance towards the vacancy in the other two directions. The tetrahedral structure is at a maximum of the BO surface along the direction of the soft modes, but a minimum along all of the other modes, placing the tetrahedral structure at a saddle point of the $\mathrm{BO}$ surface.

\section{B. Thermodynamics}

The tetragonal and pristine structures are dynamically stable at the harmonic level, and the previous section shows that the tetrahedral structure is also dynamically stable at low temperatures when anharmonicity is accounted for. Having therefore established that all three structures-tetrahedral, tetragonal, and pristine - are dynamically stable at low temperature at the anharmonic level, we turn to their thermodynamics. The static lattice, vibrational, and formation energies at $20 \mathrm{~K}$ are reported in Table I for all three structures, as at this tem- perature all three are dynamically stable. The harmonic energy $E_{\text {har }}$ and the anharmonic correction $\Delta E_{\text {anh }}=E_{\text {anh }}-E_{\text {har }}$, per atom, are given, as well as the vacancy formation energy, which is calculated as [14]

$$
E_{f}=E_{\mathrm{vac}}-\frac{N-1}{N} E_{\text {pris }},
$$

where $E_{\mathrm{vac}}$ and $E_{\text {pris }}$ are the total energies of the system with and without the vacancy, respectively, and $N$ is the number of atoms in the pristine supercell. The values of $E_{f}$ for the two different symmetry states of the vacancy are presented in the third part of Table I, at three levels of theory: static (electronic), harmonic vibrational, and anharmonic vibrational.

Because the tetrahedral state is dynamically unstable at the harmonic level (although not at the anharmonic level), due to the presence of the two soft modes, a harmonic vibrational energy cannot strictly be defined for this structure. Despite this, we have included an estimated value for the tetrahedral harmonic energy, calculated by simply cutting out the contribution of the two soft modes, to enable comparisons between the two symmetry states. The fact that this value involves the unphysical removal of two modes is noted in Table I.

With this caveat in mind, we can look at the thermodynamic stability of the two symmetry states at $20 \mathrm{~K}$. When only electronic and harmonic effects are included in the formation energy, the state with tetragonal symmetry is the most stable, although the inclusion of harmonic vibrational effects reduces the Jahn-Teller relaxation energy - the energy difference between the tetrahedral and tetragonal structures-from $0.275 \mathrm{eV}$ to $0.158 \mathrm{eV}$. Upon inclusion of anharmonic effects, the tetrahedral state becomes the most stable, as observed experimentally, by $3 \mathrm{meV}$. The predicted final formation energy for the neutral vacancy, including anharmonic effects, is therefore $8.373 \mathrm{eV}$, which is close to the estimates from experiments of 9-15 eV [50]. The formation energy of the unrelaxed vacancy at the static level is calculated to be $8.166 \mathrm{eV}$, implying a total relaxation energy of $0.989 \mathrm{eV}$ at this level of theory. (This result is not included in Table I.)

Comparing the vDoS of the vacancy structures to that of pristine diamond gives further insight into the effect of the vacancy on the vibrational properties. Figure 5(a) shows the harmonic vDoS for all three structures at high energies, with the full $\mathrm{vDoS}$ as an inset, and Fig. 5(b) shows the difference between the harmonic and anharmonic cumulative vDoS, $\Delta G(\omega)=\int_{0}^{\omega} d \omega^{\prime} g_{\text {har }}\left(\omega^{\prime}\right)-g_{\text {anh }}\left(\omega^{\prime}\right)$, for

TABLE I. DFT static lattice energies, vibrational energies, and formation energies for each structure at $20 \mathrm{~K}$. The second column shows the electronic static lattice energy $E_{\text {static }}$ per atom for the pristine, tetrahedral, and tetragonal structures relative to the pristine structure. The next two columns show the harmonic vibrational energy $E_{\text {har }}$ and anharmonic energy correction $\Delta E_{\text {anh }}=E_{\text {anh }}-E_{\text {har }}$ per atom for the three structures. The last three columns show the formation energy at the static, harmonic, and anharmonic levels of theory, $E_{f}^{\text {static }}, E_{f}^{\text {har }}$, and $E_{f}^{\text {anh }}$, respectively. The tetrahedral structure is dynamically unstable at the harmonic level, as marked by (italicized brackets).

\begin{tabular}{|c|c|c|c|c|c|c|}
\hline \multirow[b]{2}{*}{ Structure } & \multirow{2}{*}{$\frac{\text { Static energies }}{E_{\text {static }}(\mathrm{eV} / \text { atom })}$} & \multicolumn{2}{|c|}{ Vibrational energies } & \multicolumn{3}{|c|}{ Vacancy formation energies } \\
\hline & & $E_{\text {har }}(\mathrm{eV} /$ atom $)$ & $\Delta E_{\text {anh }}(\mathrm{meV} /$ atom $)$ & $E_{f}^{\text {static }}(\mathrm{eV})$ & $E_{f}^{\mathrm{har}}(\mathrm{eV})$ & $E_{f}^{\mathrm{anh}}(\mathrm{eV})$ \\
\hline Tetrahedral & 0.0292 & $(0.1826)$ & 0.190 & 7.451 & $(8.343)$ & 8.373 \\
\hline Tetragonal & 0.0281 & 0.1831 & 0.821 & 7.176 & 8.185 & 8.376 \\
\hline Pristine & 0.0000 & 0.1791 & 0.071 & 0.000 & 0.000 & 0.000 \\
\hline
\end{tabular}




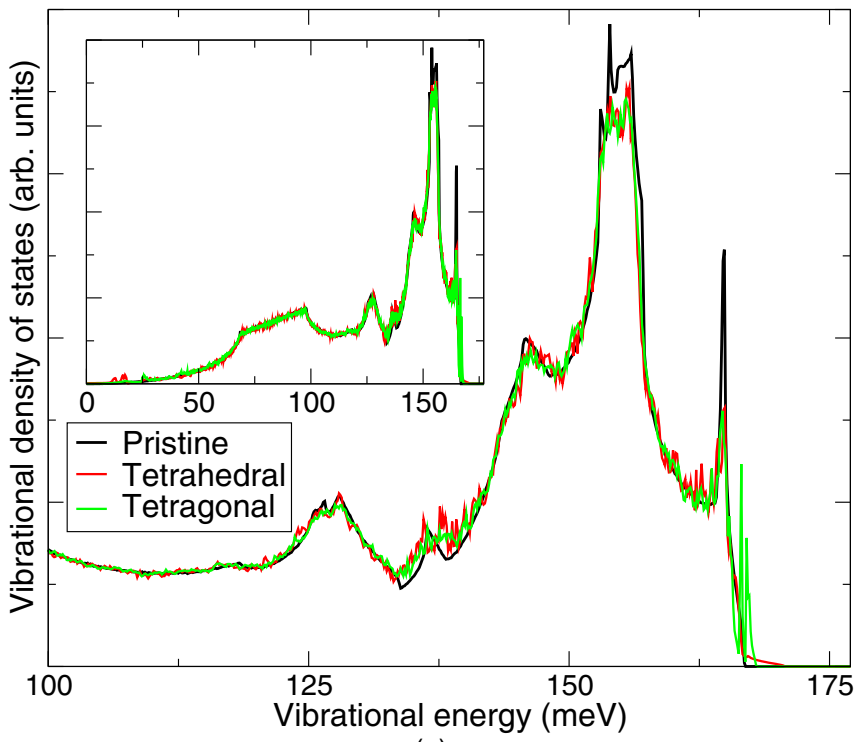

(a)

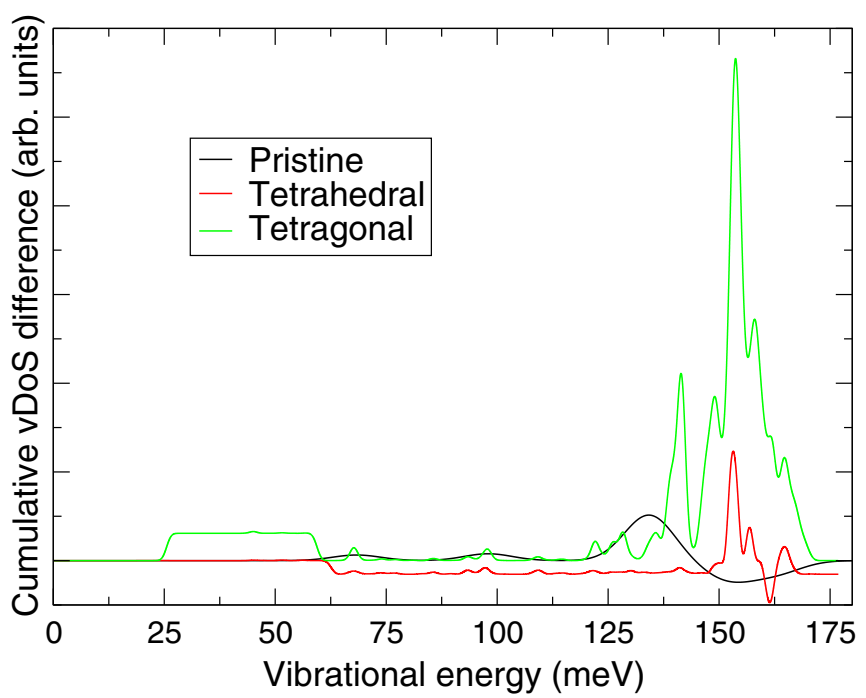

(b)

FIG. 5. (a) shows the harmonic vibrational density of states for the pristine, tetrahedral vacancy and tetragonal vacancy structures in diamond, shown at high energies above $0.1 \mathrm{eV}$ in the main plot, and in full in the inset. Note the main differences between the vacancy states and the pristine structure occur at high energies. (b) shows the difference between the harmonic and anharmonic cumulative vDoS, $\Delta G(\varepsilon)=\int_{0}^{\varepsilon} d \varepsilon^{\prime} g_{\text {har }}\left(\varepsilon^{\prime}\right)-g_{\text {anh }}\left(\varepsilon^{\prime}\right)$, of the vacancy and pristine structures, constructed using Gaussian smearing of the frequencies.

both symmetry states of the vacancy as well as the pristine lattice. The cumulative densities of states were formed by broadening the mode frequencies with Gaussians (of width $8.163 \times 10^{-4} \mathrm{eV}$ for the vacancy states and $5.442 \times 10^{-3} \mathrm{eV}$ for the pristine lattice) and cumulatively summing them. This allows us to see how the presence of anharmonicity changes the frequencies themselves. Figure 5(a) confirms that the presence of the vacancy only has a significant effect on high energy vibrations. This justifies our approach of including only the highest energy vibrational modes in the anharmonic calculations. The atoms neighboring the vacancy tend to have larger vibrational amplitudes than the other atoms in the very highest energy modes for both symmetry configurations, while for lower energy modes the amplitudes are comparable.

Figure 5(b) shows that the effect of anharmonicity is much more pronounced in the tetragonal configuration than in the tetrahedral or pristine structures. In the tetrahedral and pristine structures, the changes in the vDoS are of a similar size, and are much smaller than the changes seen in the tetragonal case. This demonstrates that distortions away from the tetrahedral symmetry of the pristine lattice strongly increase the anharmonicity of the phonon modes, with the tetrahedral vacancy retaining the weak anharmonic character of pristine diamond. The optical modes at high energies are clearly more affected by the inclusion of anharmonicity than the low-energy acoustic modes. In the pristine and tetrahedral structures, anharmonicity raises the frequency of some modes while lowering those of others, leading to both positive and negative values of $\Delta G$. In the tetragonal configuration, however, it generally raises the frequency of the modes by a small amount, showing that the leading anharmonic term is quartic in character, as cubic anharmonicity always acts to lower the energy in one dimension [35].

Given the above results at $20 \mathrm{~K}$, we briefly examine the temperature dependence of the formation energies of the tetrahedral and tetragonal structures. Neglecting the effect of thermal expansion, which is very small for diamond over the range of temperatures considered [51], we calculate the anharmonic vibrational contribution to the free energy at a set of finite temperatures, using the excited states of the VSCF Hamiltonian to construct a partition function $\mathcal{Z}$, from which we can calculate a free energy $F=-k_{B} T \ln \mathcal{Z}$ [35]. For these calculations, 80 basis functions were used to obtain accurate excited states. Figure 6 shows the anharmonic formation energy of each symmetry state of the vacancy for a range of temperatures up to $400 \mathrm{~K}$. It is clear that the tetrahedral structure remains the most stable over this temperature range; indeed, the difference in the formation energies of the two symmetry states increases from 0.003 to $0.177 \mathrm{eV}$ at $400 \mathrm{~K}$. The calculated value of the vacancy

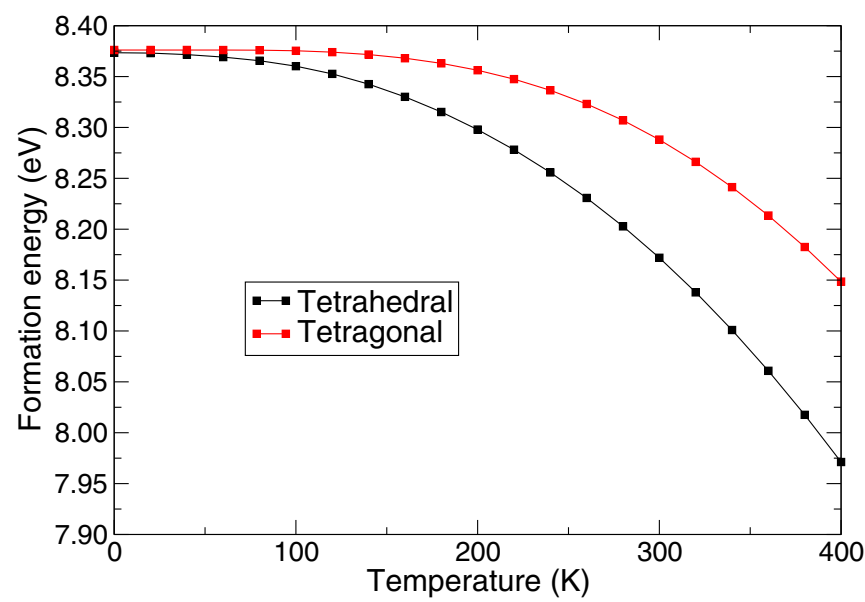

FIG. 6. Temperature dependence of the formation energies of the tetrahedral and tetragonal symmetry configurations of the neutral vacancy, including anharmonic effects but neglecting the small thermal expansion. 
formation energy at room temperature $(300 \mathrm{~K})$ is $8.172 \mathrm{eV}$, again in reasonable agreement with experimental estimates of 9-15 eV [50].

\section{CONCLUSIONS}

Our results show that the tetrahedral symmetry structure of the neutral vacancy in diamond is stabilized down to almost zero temperature by anharmonic vibrations. The vacancy undergoes a dynamic Jahn-Teller distortion which has the full $T_{d}$ point group symmetry of the pristine system, as observed experimentally. The anharmonic vibrational wave function of the tetrahedral defect has been calculated, and shown to be shared evenly among the three minima in the Born-Oppenheimer surface, which correspond to the three tetragonal distortions. We have also calculated the temperature dependence of the vacancy formation energy up to $400 \mathrm{~K}$. Our value for the formation energy of the neutral vacancy agrees well with experimental estimates of 9-15 eV [50].
Our results for the isolated neutral vacancy also imply that our method could be used to calculate anharmonic properties of other point defects, including those in diamond. Two examples of such defects are the Si-V or N-V centers, which are especially of interest due to their possible use as qubits in quantum computing [7-13]. Studies of their vibrational properties, including anharmonicity, would lead to a fuller understanding of these important defects.

\section{ACKNOWLEDGMENTS}

We thank the Engineering and Physical Sciences Research Council (EPSRC) of the UK for financial support (EP/J017639/1). B.M. acknowledges Robinson College, Cambridge, and the Cambridge Philosophical Society for a Henslow Research Fellowship. Computational resources were provided by the High Performance Computing Service at the University of Cambridge and the Archer facility of the UK's national high-performance computing service, for which access was obtained via the UKCP consortium (EP/K014560/1).
[1] A. M. Stoneham, Theory of Defects in Solids: Electronic Structure of Defects in Insulators and Semiconductors (Oxford University Press, Oxford, 1975).

[2] T. Schröder, S. L. Mouradian, J. Zheng, M. E. Trusheim, M. Walsh, E. H. Chen, L. Li, I. Bayn, and D. Englund, Quantum nanophotonics in diamond, J. Opt. Soc. Am. B 33, B65 (2016).

[3] C. Freysoldt, B. Grabowski, H. Tilmann, J. Neugebauer, G. Kresse, A. Janotti, and C. G. Van de Walle, First-principles calculations for point defects in solids, Rev. Mod. Phys. 86, 253 (2014).

[4] D. A. Drabold and S. Estreicher (eds.), Theory of Defects in Semiconductors, Topics in Applied Physics (Springer-Verlag, Berlin, 2007), Vol. 104.

[5] L. J. Rogers, K. D. Jahnke, M. W. Doherty, A. Dietrich, L. P. McGuinness, C. Müller, T. Teraji, H. Sumiya, J. Isoya, N. B. Manson, and F. Jelezko, Electronic structure of the negatively charged silicon-vacancy center in diamond, Phys. Rev. B 89, 235101 (2014).

[6] A. Gali and J. R. Maze, Ab initio study of the split siliconvacancy defect in diamond: Electronic structure and related properties, Phys. Rev. B 88, 235205 (2013).

[7] P. C. Maurer, G. Kucsko, C. Latta, L. Jiang, N. Y. Yao, S. D. Bennett, F. Pastawski, D. Hunger, N. Chisholm, M. Markham, D. J. Twitchen, J. I. Cirac, and M. D. Lukin, Room-temperature quantum bit memory exceeding one second, Science 336, 1283 (2012).

[8] L. J. Rogers, K. D. Jahnke, M. H. Metsch, A. Sipahigil, J. M. Binder, T. Teraji, H. Sumiya, J. Isoya, M. D. Lukin, P. Hemmer, and F. Jelezko, All-Optical Initialization, Readout, and Coherent Preparation of Single Silicon-Vacancy Spins in Diamond, Phys. Rev. Lett. 113, 263602 (2014).

[9] A. Sipahigil, K. D. Jahnke, L. J. Rogers, T. Teraji, J. Isoya, A. S. Zibrov, F. Jelezko, and M. D. Lukin, Indistinguishable Photons from Separated Silicon-Vacancy Centers in Diamond, Phys. Rev. Lett. 113, 113602 (2014).
[10] G. Balasubramanian, P. Neumann, D. Twitchen, M. Markham, R. Kolesov, N. Mizuochi, J. Isoya, J. Achard, J. Beck, J. Tissler, V. Jacques, P. R. Hemmer, F. Jelezko, and J. Wrachtrup, Ultralong spin coherence time in isotopically engineered diamond, Nat. Mater. 8, 383 (2009).

[11] H. Bernien, B. Hensen, W. Pfaff, G. Koolstra, M. S. Blok, L. Robledo, T. H. Taminiau, M. Markham, D. J. Twitchen, L. Childress, and R. Hanson, Heralded entanglement between solid-state qubits separated by three metres, Nature (London) 497, 86 (2013).

[12] H. S. Knowles, D. M. Kara, and M. Atatüre, Observing bulk diamond spin coherence in high-purity nanodiamonds, Nat. Mater. 13, 21 (2014).

[13] F. Dolde, I. Jakobi, B. Naydenov, N. Zhao, S. Pezzagna, C. Trautmann, J. Meijer, P. Neumann, F. Jelezko, and J. Wrachtrup, Room-temperature entanglement between single defect spins in diamond, Nat. Phys. 9, 139 (2013).

[14] F. Corsetti and A. A. Mostofi, System-size convergence of point defect properties: The case of the silicon vacancy, Phys. Rev. B 84, 035209 (2011).

[15] R. Q. Hood, P. R. C. Kent, R. J. Needs, and P. R. Briddon, Quantum Monte Carlo Study of the Optical and Diffusive Properties of the Vacancy Defect in Diamond, Phys. Rev. Lett. 91, 076403 (2003).

[16] J. Walker, Optical absorption and luminescence in diamond, Rep. Prog. Phys. 42, 1605 (1979).

[17] J. Wrachtrup and F. Jelezko, Processing quantum information in diamond, J. Phys.: Condens. Matter 18, S807 (2006).

[18] R. Schirhagl, K. Chang, M. Loretz, and C. L. Degen, Nitrogen-vacancy centers in diamond: Nanoscale sensors for physics and biology, Annu. Rev. Phys. Chem. 65, 83 (2014).

[19] G. D. Watkins, The lattice vacancy in silicon, in Deep Centers in Semiconductors, edited by Sokrates T. Pantelides (Gordon and Breach, New York, 1986). 
[20] H. A. Jahn and E. Teller, Stability of polyatomic molecules in degenerate electronic states. I. Orbital degeneracy, Proc. R. Soc. A 161, 220 (1937).

[21] M. I. J. Probert and M. C. Payne, Improving the convergence of defect calculations in supercells: An ab initio study of the neutral silicon vacancy, Phys. Rev. B 67, 075204 (2003).

[22] S. J. Breuer and P. R. Briddon, Ab initio investigation of the native defects in diamond and self-diffusion, Phys. Rev. B 51, 6984 (1995).

[23] G. Davies, The Jahn-Teller effect and vibronic coupling at deep levels in diamond, Rep. Prog. Phys. 44, 787 (1981).

[24] C. D. Clark and J. Walker, The neutral vacancy in diamond, Proc. R. Soc. A 334, 241 (1973).

[25] M. Lannoo and A. M. Stoneham, The optical absorption of the neutral vacancy in diamond, J. Phys. Chem. Solids 29, 1987 (1968).

[26] F. S. Ham, The Jahn-Teller effect, Int. J. Quantum Chem. 5, 191 (1971).

[27] A. J. Millis, B. I. Shraiman, and R. Mueller, Dynamic JahnTeller Effect and Colossal Magnetoresistance in $\mathrm{La}_{1-x} \mathrm{Sr}_{x} \mathrm{MnO}_{3}$, Phys. Rev. Lett. 77, 175 (1996).

[28] V. Dediu, C. Ferdeghini, F. C. Matacotta, P. Nozar, and G. Ruani, Jahn-Teller Dynamics in Charge-Ordered Manganites from Raman Spectroscopy, Phys. Rev. Lett. 84, 4489 (2000).

[29] V. Brouet, H. Alloul, T. N. Le, S. Garaj, and L. Forró, Role of Dynamic Jahn-Teller Distortions in $\mathrm{Na}_{2} \mathrm{C}_{60}$ and $\mathrm{Na}_{2} \mathrm{CsC}_{60}$ Studied by NMR, Phys. Rev. Lett. 86, 4680 (2001).

[30] S. E. Canton, A. J. Yencha, E. Kukk, J. D. Bozek, M. C. A. Lopes, G. Snell, and N. Berrah, Experimental Evidence of a Dynamic Jahn-Teller Effect in $\mathrm{C}_{60}^{+}$, Phys. Rev. Lett. 89, 045502 (2002).

[31] M. C. M. O'Brien, The dynamic Jahn-Teller effect in octahedrally co-ordinated d ${ }^{9}$ ions, Proc. Roy. Soc. A 281, 323 (1964).

[32] K.-M. C. Fu, C. Santori, P. E. Barclay, L. J. Rogers, N. B. Manson, and R. G. Beausoleil, Observation of the Dynamic Jahn-Teller Effect in the Excited States of Nitrogen-Vacancy Centers in Diamond, Phys. Rev. Lett. 103, 256404 (2009).

[33] A. M. Stoneham, The low-lying levels of the GR 1 center in diamond, Solid State Commun. 21, 339 (1977).

[34] R. O. Jones, Density functional theory: Its origins, rise to prominence, and future, Rev. Mod. Phys. 87, 897 (2015).

[35] B. Monserrat, N. D. Drummond, and R. J. Needs, Anharmonic vibrational properties in periodic systems: Energy, electronphonon coupling, and stress, Phys. Rev. B 87, 144302 (2013).

[36] S. J. Clark, M. D. Segall, C. J. Pickard, P. J. Hasnip, M. I. J. Probert, K. Refson, and M. C. Payne, First principles methods using CASTEP, Z. Kristallogr. 220, 567 (2005).
[37] D. Vanderbilt, Soft self-consistent pseudopotentials in a generalized eigenvalue formalism, Phys. Rev. B 41, 7892 (1990).

[38] J. P. Perdew and A. Zunger, Self-interaction correction to density-functional approximations for many-electron systems, Phys. Rev. B 23, 5048 (1981).

[39] O. K. Al-Mushadani and R. J. Needs, Free-energy calculations of intrinsic point defects in silicon, Phys. Rev. B 68, 235205 (2003).

[40] K. Kunc, I. Loa, and K. Syassen, Equation of state and phonon frequency calculations of diamond at high pressures, Phys. Rev. B 68, 094107 (2003).

[41] R. Maezono, A. Ma, M. D. Towler, and R. J. Needs, Equation of State and Raman Frequency of Diamond from Quantum Monte Carlo Simulations, Phys. Rev. Lett. 98, 025701 (2007).

[42] O. Madelung, (ed.), Semiconductors: Basic Data (Springer, Berlin, 1996).

[43] H. J. Monkhorst and J. D. Pack, Special points for Brillouin-zone integrations, Phys. Rev. B 13, 5188 (1976).

[44] K. Kunc and R. M. Martin, Ab initio Force Constants of GaAs: A New Approach to Calculation of Phonons and Dielectric Properties, Phys. Rev. Lett. 48, 406 (1982).

[45] S. Azadi, B. Monserrat, W. M. C. Foulkes, and R. J. Needs, Dissociation of High-Pressure Solid Molecular Hydrogen: A Quantum Monte Carlo and Anharmonic Vibrational Study, Phys. Rev. Lett. 112, 165501 (2014).

[46] B. Monserrat, N. D. Drummond, Chris J. Pickard, and R. J. Needs, Electron-Phonon Coupling and the Metallization of Solid Helium at Terapascal Pressures, Phys. Rev. Lett. 112, 055504 (2014).

[47] E. A. Engel, B. Monserrat, and R. J. Needs, Anharmonic Nuclear Motion and the Relative Stability of Hexagonal and Cubic Ice, Phys. Rev. X 5, 021033 (2015).

[48] J. O. Jung and R. B. Gerber, Vibrational wave functions and spectroscopy of $\left(\mathrm{H}_{2} \mathrm{O}\right)_{n}, n=2,3,4,5$ : Vibrational selfconsistent field with correlation corrections, J. Chem. Phys. 105, 10332 (1996).

[49] See Supplemental Material at http://link.aps.org/supplemental/ 10.1103/PhysRevB.95.014108 for details of the pseudopotential used, the $T_{d}$ equilibrium structure and soft mode displacement patterns, and further cuts through the Born-Oppenheimer surface shown in Fig. 4.

[50] J. C. Bourgoin, An experimental estimation of the vacancy formation energy in diamond, Radiat. Eff. Defects Solids 79, 235 (1983).

[51] S. Stoupin and Y. V. Shvyd'ko, Thermal Expansion of Diamond at Low Temperatures, Phys. Rev. Lett. 104, 085901 (2010). 